\title{
Correction to Fully Enclosed Microfluidic Paper-Based Analytical Devices
}

Kevin M. Schilling, Anna L. Lepore, Jason A. Kurian, and Andres W. Martinez*

Anal. Chem. 201284 (3), 1579-1585. DOI: 10.1021/ac202837s

There is an error in the units of the concentrations of potassium iodide and trehalose described in the experimental details on page 1581 . The correct concentrations are $0.6 \mathrm{M}$ potassium iodide and $0.3 \mathrm{M}$ trehalose. 